\title{
Comparative Electrochemical Investigation of Pt, Au and Ti Electrodes on Liquid Crystal Polymer for the Application of Neuromuscular Prostheses
}

\author{
S. Mohtashami, M. R. Howlader ${ }^{*}$, and T. E. Doyle**, \\ Department of Electrical and Computer Engineering, McMaster University, Hamilton, \\ Ontario L8S 4K1, Canada \\ *Email: mrhowlader@ece.mcmaster.ca \\ **Email: doylet@mcmaster.ca
}

This paper studies the electrochemical impedance of Platinum $(\mathrm{Pt})$, Gold ( $\mathrm{Au}$ ), and Titanium (Ti) deposited on liquid crystal polymer (LCP) to understand charge transfer mechanism of implantable electrodes for neuromuscular electrical stimulation. Electrodes are fabricated through e-beam evaporation and characterized using electrochemical impedance spectroscopy (EIS) and atomic force microscopy (AFM) measurements. A theoretical model is proposed to explain physical functionalities. The results demonstrate as the size of electrodes increases, higher conductivity and double layer capacitance are obtained. In neuromuscular stimulation frequencies, Pt electrodes offer the best conductivity followed by $\mathrm{Ti}$ and $\mathrm{Au}$ respectively. This material dependence of impedance magnitude is related to the surface morphology of the electrodes and is assessed by AFM. Deposition pressure dependence of Ti electrodes on the impedance is observed due to change of surface roughness and double layer capacitor. This study addresses the interface impedance of implanted electrodes and permits further development of neuromuscular prostheses.

\section{Introduction}

There are many muscle control disorders due to the absence or failure of neural impulses reaching their natural destination. While peripheral nerves and muscles are still intact, the patient does not have the ability to activate them voluntarily. Intact neurons can be activated via the electrodes by external signals through electrical stimulation. Therefore, electrical stimulation has been widely utilized by many research groups as the basis of emerging prostheses and treatments for spinal cord injuries, stroke, sensory deficits and neurological disorders (1), for control of respiration, bowel and bladder, restoring vision sense at outer retina and providing partial hearing (2-5).

The electrical stimulation techniques require a means of generating an electric pulse, a way to route the pulse to the nerve, and electrodes to deliver an effective stimulus to the nerve to activate the target tissue. These techniques share common design constraints that include: lower power consumption, small size, and the capability of effective and safe stimulation (6). To accomplish effective and safe stimulation, the designer must balance the amount of stimulus current (high) against the device size and power (increased with higher current). The area to optimize these requirements is the stimulating electrode. Stimulating electrodes, if understood, fabricated and characterized precisely, could offer 
more efficient electrical stimulation, with low power consumption and minimal, if any, electrode-tissue interface injuries.

Compared to other methods of electrical stimulation such as transcutaneous and percutaneous stimulation, implanted electrodes provide better selectivity, lower stimulation current intensity, and reduced rate of fatigue (7). However, the primary challenge of designing an implanted electrode has always been the biocompatibility of the electrode in contact with host tissue (8-10). Biocompatibility involves avoiding toxic or immune responses in the adjacent tissue or medium. Electrode materials such as platinum $(\mathrm{Pt})$, gold $(\mathrm{Au})$, titanium $(\mathrm{Ti})$, iridium (Ir), iridium oxide $\left(\mathrm{IrO}_{2}\right)$, and carbon $(\mathrm{C})$ have been studied extensively and are reported to be well tolerated by the tissue with no toxicity concern (10-12). However, for $\mathrm{Ir}$ and $\mathrm{IrO}_{2}$ stimulation electrodes, in vivo delamination is reported (1).

Beyond their biocompatibility, implanted electrodes require careful consideration of electrochemical conductivity. In particular, material, size, and deposition conditions should be taken into account because these parameters control the performance of electrodes, therefore, are very important to study in achieving a safer and efficient stimulation. In the case of biomaterial evaluations, nanoporous carbon is reported to dominate stainless steel, titanium nitride (TiN), and $\mathrm{Ti}$ in terms of electrochemical conductivity and stability (10). However, poor mechanical strength and difficulty in handling of nanoporous carbon limit its application. TiN is suggested as a more compatible film with the microfabrication methods, and is evaluated compared with $\mathrm{IrO}_{2}$ $(1,4)$. Despite good charge transfer parameters of $\mathrm{TiN}, \mathrm{IrO}_{2}$ electrochemically dominates (4). $\mathrm{IrO}_{2}, \mathrm{Pt}$, and $\mathrm{Au}$ are demonstrated to have high charge transfer parameters and corrosion resistance on rigid substrates of silicon (9).

Recently, polymer substrates have attracted much attention due to their flexibility, biocompatibility, and mechanical strength (9,11-12). Flexible substrates enhance mechanical fixation and provide a safer, more efficient excitation by increasing the charge transfer at electrode/tissue interface. Polyimide (PI) is a common flexible substrate in electrical stimulation electrodes (10-14). However, it has high water absorption (15). Water absorption causes hydrolytic attack of the substrate material that causes delamination of deposited electrodes through adhesion layer/polyimide interface. To overcome this challenge, we present liquid crystal polymer (LCP) as the flexible substrate material in this study. To the best of our knowledge, there is no comparative evaluation of LCP substrates in neuromuscular stimulation electrodes. Compared to other flexible substrates, such as polyimide, LCP has very little water absorption (15), which provides better electrochemical stability of electrodes for long term applications.

$\mathrm{Pt}, \mathrm{Au}$, and Ti electrodes of different surface areas were deposited at different base pressures on LCP to find the one with best charge transfer parameters. Electrodes were fabricated in four circular surface areas to evaluate the effect of electrode size on electrode-tissue impedance. Electrochemical investigation of deposited films in the biological environment was performed by electrochemical impedance spectroscopy (EIS). The EIS measurements were performed at room temperature with 0.1-molar phosphate buffered saline (PBS) solution. PBS is a saline solution containing sodium chloride, sodium phosphate, potassium phosphate and potassium chloride, and is commonly used as the physiological medium in biological research $(10-12,16)$. Root mean square (RMS) surface roughness, Bode plots, and the equivalent circuit model of the electrode/electrolyte interface of the three electrode types were studied to evaluate the electrodes charge transfer parameters and to research their usefulness in different neuromuscular prosthetic applications. 


\section{Experimental}

\section{Materials}

For the development of highly flexible nerve electrodes, LCP was selected for the substrate material. Materials to be considered for use as the electroactive portion of a neural stimulation electrode must accomplish stimulation without causing neural injury. To avoid neural injuries, stimulation electrodes need to have the capability to transfer the required coulomb charge without causing electrode corrosion or electrolyzing the tissue. The electrochemical conductivity of electrodes depends on the surface area of electrodetissue interface. Hence, electrode material and its deposition technique determine the size of electrodes regarding its charge injection capacity. $\mathrm{Pt}, \mathrm{Au}$, and $\mathrm{Ti}$ are served as conducting materials that offer well-established biocompatibility and fulfill these requirements. For the deposition of $\mathrm{Pt}, \mathrm{Au}$ and $\mathrm{Ti}$ films, the purities were $99.99 \%$, $99.999 \%$ and $99.99 \%$ respectively.

\section{Electrodes Fabrication}

Electrodes with circular shapes are fabricated, as shown in figure 1, on LCP substrate with $50 \mu \mathrm{m}$ thickness. Tails were designed to provide a contact out of the electrodes without affecting its surface. After wire connection, tails were covered by insulating glue so that only circular spaces are in effect for charge transfer. To enhance the adhesion of the conducting layer to LCP substrate, a $30 \mathrm{~nm}$ Ti layer was deposited between the conducting layer and LCP. Pt, Au, and Ti are served as conducting materials with $200 \mathrm{~nm}$ thickness. A $0.1 \mathrm{~mm}$ thick copper mask prepared by water jetting technique with sharp circular pores of $3,4,6$, and $8 \mathrm{~mm}$ diameter was placed on the LCP substrate. Adhesion and conducting layers were deposited through electron beam evaporation technique at deposition rate of 1.5 Angstroms/second with background pressure of 1E-6 Torr. To investigate the effect of deposition pressure on electrodes performance, another set of $\mathrm{Ti}$ samples was deposited using the same technique and parameters but at a different background pressure of 3E-8 Torr.

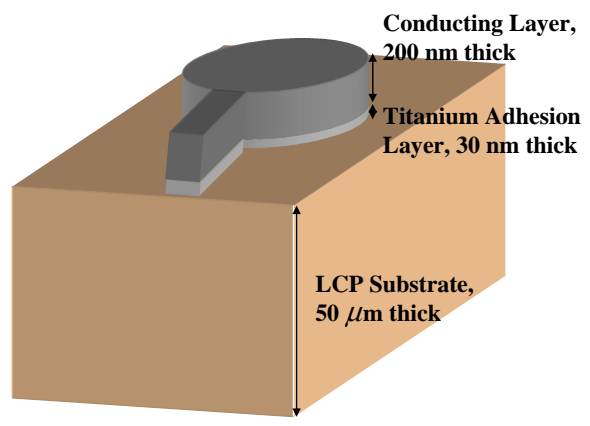

Figure 1. Schematic diagram of a deposited electrode on the LCP substrate.

\section{$\underline{\text { Characterization }}$}

Prepared electrodes were then electrochemically investigated using electrochemical impedance spectroscopy (EIS). Their RMS surface roughness was measured by atomic force microscopy (AFM) technique. All electrochemical measurements were 
implemented in a 0.1-molar phosphate buffered saline (PBS) solution at room temperature. A standard, three-electrode-cell set up was used as the configuration. Figure 2 shows a schematic of the measurements set up, with the equivalent circuit that models the electrode/electrolyte interface. The equivalent circuit of the system consists of an interface constant phase element (CPE) with capacitive behavior modeling the double layer, in parallel with a charge transfer resistance $\left(\mathrm{R}_{\mathrm{ct}}\right)$ together in series with the electrolyte resistance $\left(R_{e}\right)$. The current flow through the LCP substrate was neglected because of its high resistance compared to that of electrodes. Pt, $\mathrm{Au}$, and Ti samples were served as the working electrodes in each test. A calomel reference electrode was immersed in an electrolytic solution in a glass container. A platinum wire was served as the counter electrode with a glass rod as the support in the cell. An electrochemical workstation with Gamry Framework was used to acquire the impedance spectra of the Pt, $\mathrm{Au}$, and Ti electrodes. For EIS measurements, the alternating current excitation voltage was $10 \mathrm{mV}$ at a frequency range from $0.1 \mathrm{~Hz}$ to $100 \mathrm{KHz}$. Equivalent circuits and parameters were determined with ZPlot software.

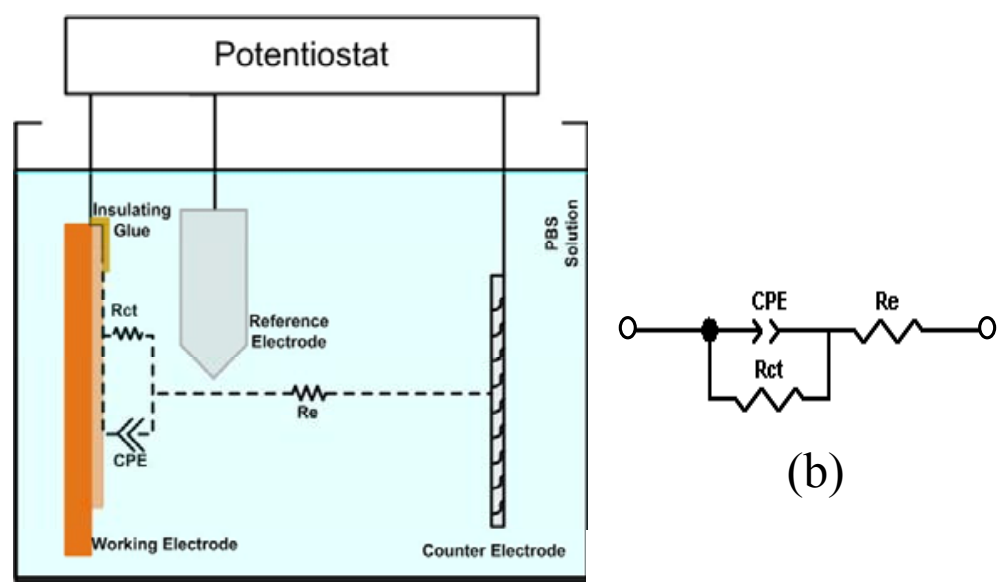

(a)

Figure 2. (a) Schematic of electrochemical measurements set up showing the equivalent circuit model, and (b) the equivalent circuit model formed at the electrode/electrolyte interface.

From the model circuit, considering $\mathrm{CPE}$ as an ideal capacitor, shown as $\mathrm{C}_{\mathrm{dl}}$, its impedance is presented as:

$$
Z(j \omega)=\left(R_{e}+R_{c t}\right) \frac{1+j \omega\left(\frac{R_{e} R_{c t} C_{d l}}{R_{e}+R_{c t}}\right)}{1+j \omega\left(R_{c t} C_{d l}\right)}=\left(R_{e}+R_{c t}\right) \frac{1+j \omega \tau_{2}}{1+j \omega \tau_{1}}
$$

At high frequencies, the effect of $R_{c t}$ may be neglected because the system functions like a high-pass filter. The cut off frequency of this high-pass filter, which is defined as the frequency at the interception point of rising slope tangent with the line of constant impedance, is obtained by:

$$
f_{c}=\frac{1}{2 \pi R_{e} C_{d l}}
$$


At very high frequencies, $\mathrm{C}_{\mathrm{dl}}$ dominates and the resulting interface impedance is approximately the electrolyte resistance, $\mathrm{R}_{\mathrm{e}}$, in series with $\mathrm{C}_{\mathrm{dl}}$. At very low frequencies, $\mathrm{C}_{\mathrm{dl}}$ becomes an open circuit, resulting in an equivalent impedance of $\mathrm{R}_{\mathrm{e}}+\mathrm{R}_{\mathrm{ct}}$. On the other hand, the value of $\mathrm{C}_{\mathrm{dl}}$ is proportional to the surface area $\left(C_{d l} \propto A\right)$. Since an electrode with a rougher surface exhibits a larger surface area, $C_{d l}$ increases with the increase of surface roughness. Therefore, an electrode with a rougher surface would exhibit a lower cut off frequency. However, double layer is not a pure capacitor but a constant phase element with $\beta$ deviation from pure capacitive behavior and is calculated as equation 3 .

$$
Z_{C P E}=\frac{1}{K(j \omega)^{\beta}}
$$

$\mathrm{K}$ is magnitude and $\beta$ a constant between 0 and 1 , where $\beta=1$ shows an ideal capacitor and $\beta=0$ shows a pure resistor. Due to capacitive behavior of the double layer, the value of $\beta$ is desired to be close to 1 . These model parameters provide information about each individual interfacial process.

To validate the measured experimental model parameters, the RMS surface roughness values of electrodes are compared using AFM. All measured RMS surface roughness values are averaged from scanning three different areas of each electrode with standard deviation of less than $10 \%$ of each raw value.

\section{Results}

\section{Measurement of Surface Roughness}

Table I shows the RMS surface roughness value of each film. As mentioned earlier, higher surface roughness increasing the real surface area results in bigger values of double layer capacitance. According to equation 2, higher values of double layer

TABLE I. RMS values of surface roughness of all samples obtained from AFM. Au offers the smoothest surface followed by Pt and Ti respectively. Lower deposition pressure increases the surface roughness of Ti films.

\begin{tabular}{lcc}
\hline Films & $500 \times 500 \mathrm{~nm}^{2}$ & $1 \times 1 \mu^{2}$ \\
\hline Au on LCP & $2.12 \mathrm{~nm}$ & $3.18 \mathrm{~nm}$ \\
Pt on LCP & $2.87 \mathrm{~nm}$ & $3.44 \mathrm{~nm}$ \\
Tí on LCP & $3.58 \mathrm{~nm}$ & $4.49 \mathrm{~nm}$ \\
$\begin{array}{l}\text { Ti on LCP- } \\
\text { lower pressure }\end{array}$ & $4.93 \mathrm{~nm}$ & $5.49 \mathrm{~nm}$
\end{tabular}

capacitance decrease the cut off frequency. The location of $f_{c}$ together with the value of $\beta$, which sets the slope of conductivity spectra, define the conductivity in low frequencies, 
and hence are significant to assess. The data demonstrates that Au electrodes offer the smoothest surface followed by $\mathrm{Pt}$ and $\mathrm{Ti}$, respectively. Ti films deposited under lower pressure have rougher surfaces. Therefore, Au electrodes are expected to exhibit the highest $f_{c}$, followed by $\mathrm{Pt}, \mathrm{Ti}$, and Ti deposited at lower frequencies, which is validated in figure 3. Figure 3 is derived from the electrodes EIS magnitude spectra. It demonstrates cut off frequencies of each electrode at all diameter sizes.

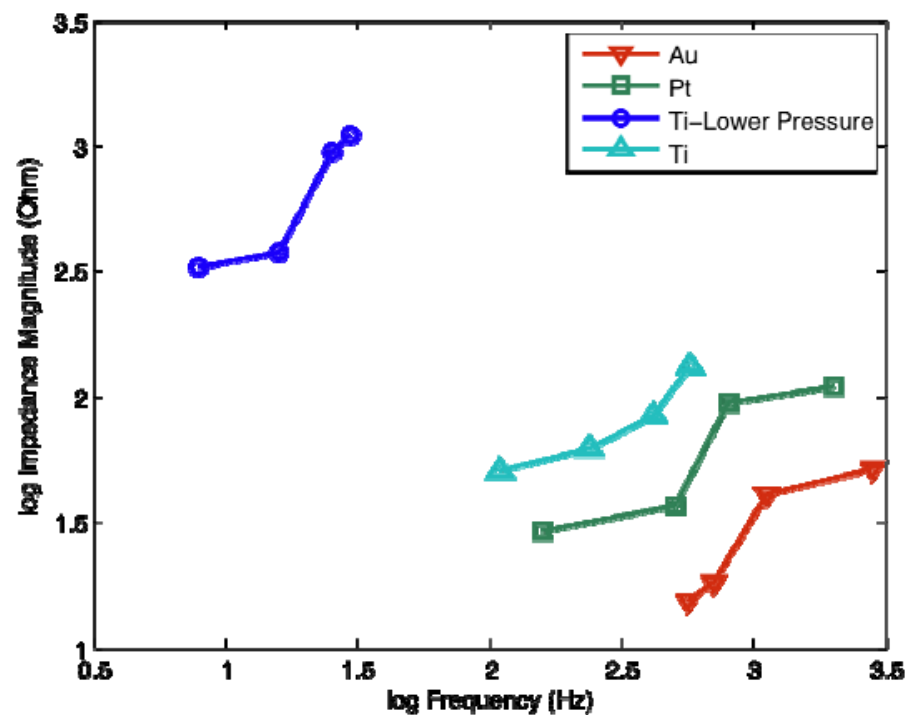

Figure 3. Quantitative look at the cut off frequencies of each film at all sizes. Bigger sizes of a single electrode have higher values of $\mathrm{C}_{\mathrm{dl}}$ which shifts the cut off frequencies toward lower frequencies. Electrodes with rougher materials have higher values of $\mathrm{C}_{\mathrm{dl}}$ which shifts the cut off frequencies toward lower frequencies.

\section{Size Dependence of Electrodes' Impedance}

Figure 4 shows the Bode plots of measured EIS from Pt, Au, and Ti films deposited under the same conditions. For each electrode, as its area diameter increases, the impedance magnitude decreases at all frequencies. The identical effect of electrode area on impedance is reported for Au electrodes on PI substrates (12). The values of the circuit model parameters obtained by fitting the impedance experimental data to the equivalent circuit model are shown in Table II. It is inferred that with the decrease of electrode area, the magnitude of CPE (K) decreases while $R_{c t}$ and $R_{e}$ increase. We do not observe any $\beta$ dependence of size in the results. Hung et. al (17) and Brett (18) also mentioned the same relation of electrochemical biosensors size with $\mathrm{K}$ and $\mathrm{R}_{\mathrm{ct}}$. As shown in figure 3 and discussed earlier, electrodes with larger surface areas, offer lower cut off frequencies due to their higher magnitudes of CPE.

\section{Material Dependence of Electrodes Impedance}

Figure 5 shows a comparison of impedance magnitude of different materials with the same sizes. The important difference in impedance spectra of these metals in neuromuscular stimulation frequency range, is the location of $f_{c}$ and value of $\beta$. 

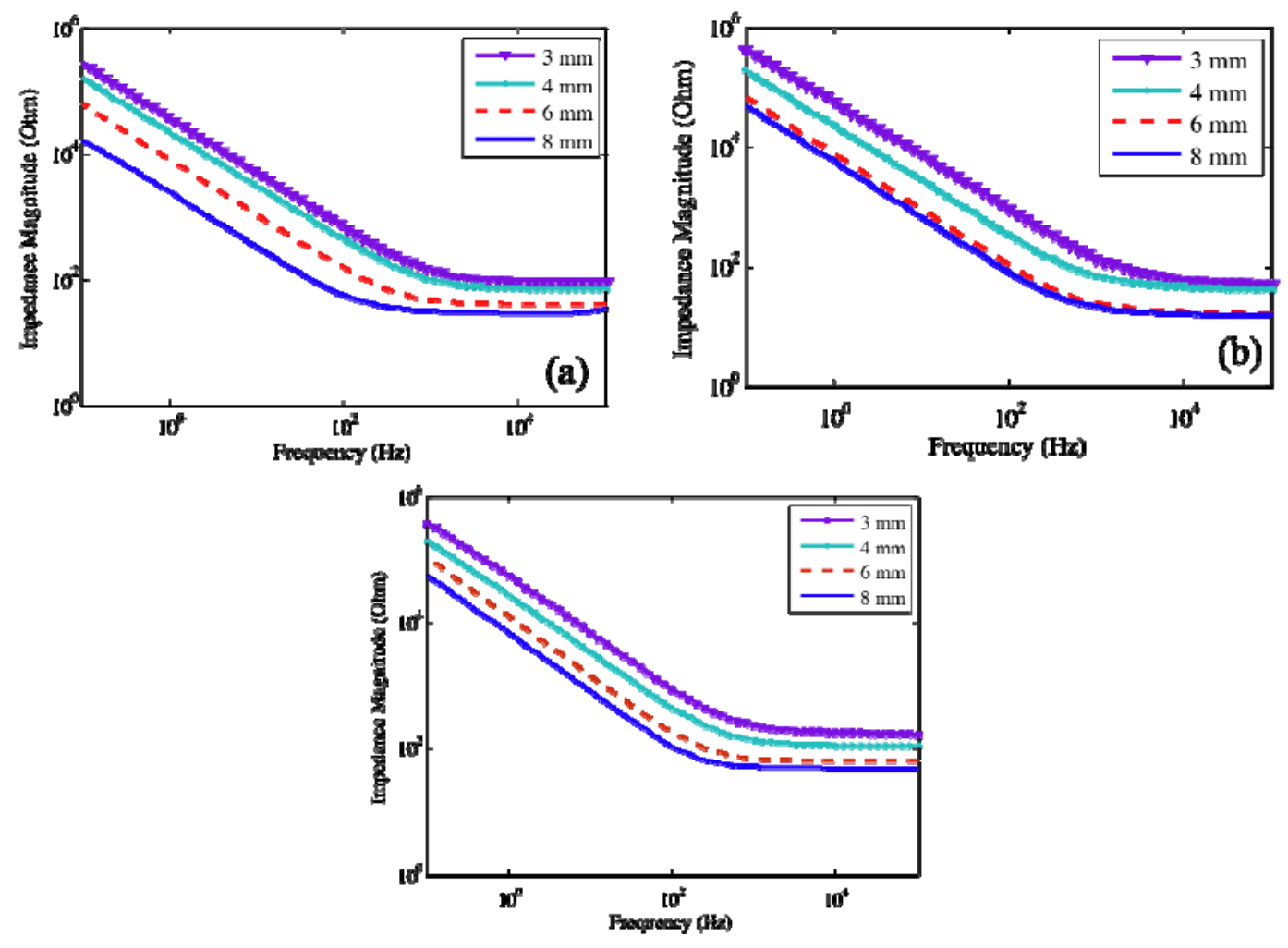

Figure 4. Size dependent electrochemical impedance of (a) Pt, (b) $\mathrm{Au}$ and (c) $\mathrm{Ti}$ electrodes. Impedance decreases with increasing area of electrodes.
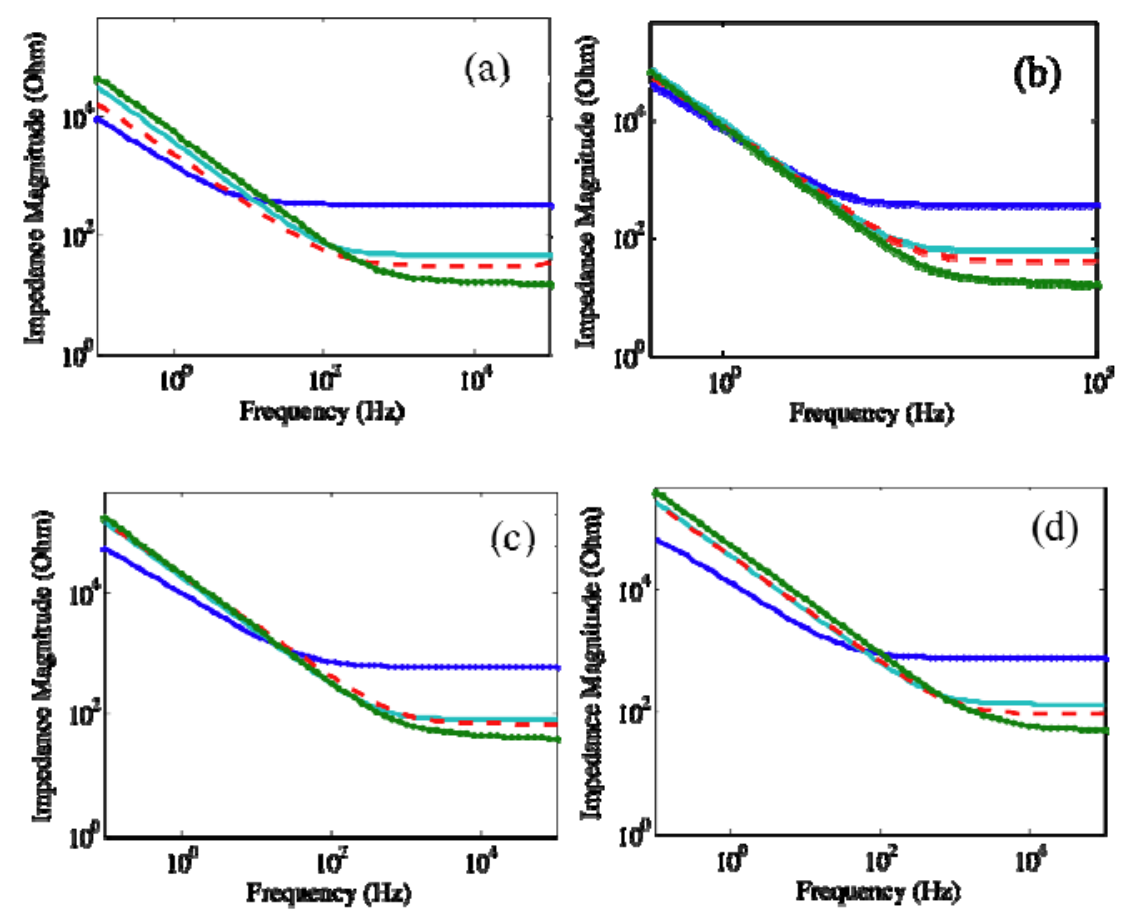

- Ti-deposited at lower pressure

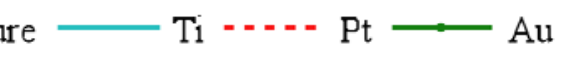

Figure 5. Comparison of impedance of $\mathrm{Au}, \mathrm{Pt}$, and $\mathrm{Ti}$ electrodes at different deposition pressures and diameter: (a) 8, (b) 6, (c) 4, and (d) $3 \mathrm{~mm}$ diameter size. * indicates desired frequency range for neuromuscular stimulation. 
At frequencies lower than its $\mathrm{f}_{\mathrm{c}}$, each electrode shows the capacitive behavior with a rising slope of $-\beta$, as discussed in equations 1 and 2. Compared to $\mathrm{Pt}$ and $\mathrm{Ti}, \mathrm{Au}$ has the highest $f_{c}$ due to its smoothest surface. This high $f_{c}$ along with its high value of $\beta$ provides $\mathrm{Au}$ with highest impedance magnitude in frequencies lower than its $\mathrm{f}_{\mathrm{c}}$. Despite higher $\mathrm{f}_{\mathrm{c}}$ of Pt compared to Ti, it proposes lower impedance which is because of its lower $\beta$ values. However, in neuromuscular stimulation frequency range, $\mathrm{Pt}$ and Ti with similar values, offer the highest conductivity. From Tandon et. al study (10), it is also observed that similar to our study, the material with largest magnitude of CPE, demonstrates the lowest cut off frequency in EIS spectra. In addition, the main shape of EIS spectra is alike for all the measured materials.

\section{Deposition Pressure}

For those Ti samples deposited under lower pressure of 3E-8 Torr, having rougher surfaces (see table I), higher magnitudes of CPE are expected as confirmed by table II. These low pressure deposited Ti electrodes with larger double layer capacities offer lower cut off frequencies and $\beta$ values, compared to the Ti films deposited under higher pressure of 1E-6 Torr. This results in their higher conductivity in low frequencies that are of our interest.

TABLE II. The model parameters taken from curve fitting of experimental data to the proposed model. Strong dependence of CPE and $\beta$ on the diameter and type of electrode materials are observed.

\begin{tabular}{|c|c|c|c|c|c|}
\hline Material & Area dihmeter & $R_{8}(\mathbf{Q})$ & |CPE| & $\beta$ & $\mathbf{R}_{\mathrm{gs}}(\mathbf{M} \mathbf{\Omega})$ \\
\hline \multirow{4}{*}{$\mathrm{Au}$} & $8 \mathrm{~mm}$ & 17.85 & $3.0 \mathrm{E}-5 \mathrm{~A}$ & 0.92 & 0.25 \\
\hline & $6 \mathrm{~mm}$ & 19.07 & $1.8 \mathrm{E}-5$ & 0.93 & 1.2 \\
\hline & $4 \mathrm{~mm}$ & 48.87 & $7.8 \mathrm{E}-6$ & 0.91 & 2.85 \\
\hline & $3 \mathrm{~mm}$ & $49.19 \downarrow$ & $3.3 \mathrm{E}-6$ & 0.88 & $5.08 \downarrow$ \\
\hline \multirow[t]{4}{*}{$\mathbf{P t}$} & $8 \mathrm{~mm}$ & 34 & 8.1 E-5 & 0.89 & 2.1 \\
\hline & $6 \mathrm{~mm}$ & 51 & $2.1 \mathrm{E}-5$ & 0.88 & 2.7 \\
\hline & $4 \mathrm{~mm}$ & 70 & $9.5 \mathrm{E}-6$ & 0.86 & 3 \\
\hline & $3 \mathrm{~mm}$ & $90 \downarrow$ & $5.5 \mathrm{E}-6$ & 0.87 & $3 \downarrow$ \\
\hline \multirow{4}{*}{$\mathbf{T i}$} & $8 \mathrm{~mm}$ & 55.2 & $5.2 \mathrm{E}-5 \mathrm{~A}$ & 0.93 & 2.4 \\
\hline & $6 \mathrm{~mm}$ & 61.6 & $2.2 \mathrm{E}-5$ & 0.93 & 2.4 \\
\hline & $4 \mathrm{~mm}$ & 67.9 & $1.1 \mathrm{E}-5$ & 0.90 & 2.5 \\
\hline & $3 \mathrm{~mm}$ & $131.7 \checkmark$ & $5.7 \mathrm{E}-6$ & 0.89 & $3.6 \downarrow$ \\
\hline \multirow{4}{*}{$\begin{array}{l}\text { Ti- lower } \\
\text { pressure }\end{array}$} & $8 \mathrm{~mm}$ & 326 & $1.5 \mathrm{E}-4 \mathrm{P}$ & 0.84 & 103 \\
\hline & $6 \mathrm{~mm}$ & 351.1 & $1.9 \mathrm{E}-4$ & 0.70 & 10.8 \\
\hline & $4 \mathrm{~mm}$ & 3702 & $1.8 \mathrm{E}-4$ & 0.72 & 10.9 \\
\hline & $3 \mathbf{m m}$ & $383.7 \checkmark$ & $1.8 \mathrm{E}-4$ & 0.70 & $11 \downarrow$ \\
\hline
\end{tabular}

\section{Discussion}

Experimental results reveal material dependence of impedance magnitude is due to the cut off frequency and $\beta$ value that each material presents. Cut off frequencies shift toward lower frequencies with the increase of the double layer capacitance and decrease of the deposition pressure. This phenomenon is related to the surface morphology, structural 
quality and deposition parameters of the electrodes. The AFM evaluations demonstrate that $\mathrm{Au}$ electrodes offer the smoothest surface followed by Pt and Ti respectively. Ti films deposited under lower pressure have rougher surfaces. Higher values of surface roughness provide bigger real surface areas resulting in bigger values of double layer capacitance. Hence, according to equation 2, cut off frequency is shifted toward lower frequencies leading toward better conductivity in lower frequency range. However, compared to $\mathrm{Ti}, \mathrm{Pt}$ electrodes with higher $\mathrm{f}_{\mathrm{c}}$ are proposed for implantable stimulation electrodes due to their lower impedance related to lower $\beta$ values.

\section{Perspective Applications}

The perspective application for the implantable and flexible stimulation electrode is the neuromuscular prosthetic system as presented in figure 6. Such systems are becoming technologically feasible with advances in brain-computer-interfaces (BCI) (19-21).

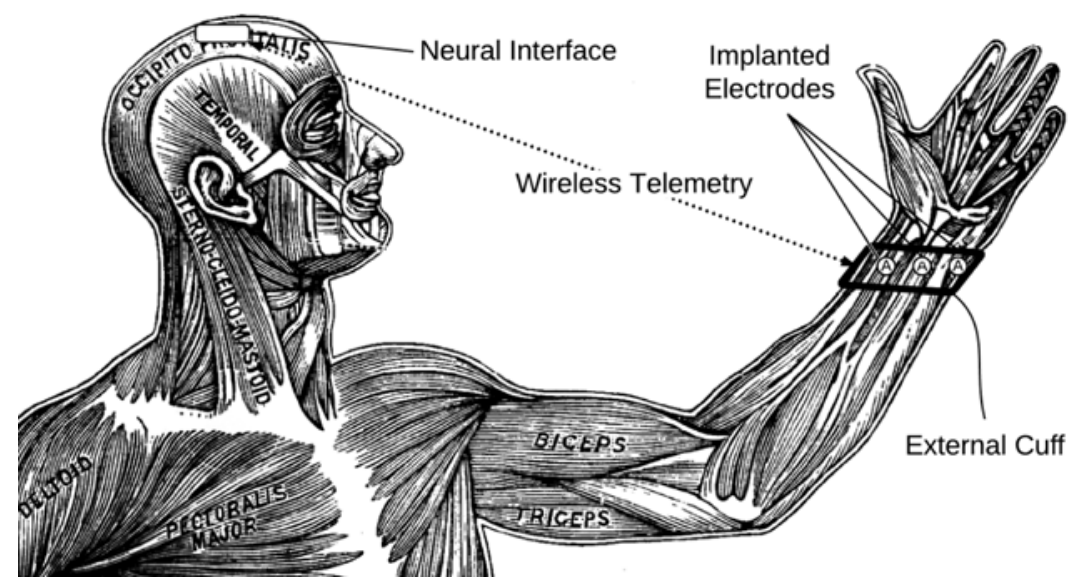

Figure 6. Proposed Neuromuscular Prosthetic System. Line art courtesy of FCIT.

A primary limiting factor in such systems is the interface of the implanted hardware to the biological system. While a "traditional" prosthesis replaces a limb, our system seeks to assist subjects that have weakness of loss of normal control due to the interruption of normal efferent and/or afferent signals. The proposed system stimulates the nerves that activate the muscular contraction that will require less activation current than electrically activating the muscle directly.

The neural interface is normally implemented using specific montages for ambulatory BCI. The result is fewer electrodes compared to a standard electroencephalography 10-20 electrode arrangement. The challenge with a scalp surface mounted neural interface is the accuracy of control due to both physiological and environmental noise. An emerging method of neural interfacing with high accuracy and fidelity is performed through electrocorticography (ECoG) (20-22). This method is highly invasive and will require better (less invasive) implementation along with an electrode system that has been carefully designed for implantation.

With the presented electrode design, the authors believe they have provided a solution to the primary challenge of interface impedance to the biological system through flexible LCP substrate with deposited electrode materials to focus the range of stimulation frequencies. 


\section{Conclusion}

Flexible electrodes on LCP substrate have been fabricated and electrochemically investigated in this study. $\mathrm{Pt}, \mathrm{Ti}$, and $\mathrm{Au}$ were deposited as conducting materials in different surface areas. A set of Ti electrodes was prepared at a different deposition pressure. The results have proved that as the size of electrodes is increased, their impedance magnitude decreases and they offer higher double layer capacitance. In neuromuscular stimulation frequency range, platinum electrodes offer the best conductivity followed by titanium and gold respectively, while in higher frequencies, $\mathrm{Au}$ dominates $\mathrm{Pt}$ and $\mathrm{Ti}$. This result may permit better design and miniaturization of electrochemical electrodes for electrical stimulation of neural prostheses.

\section{Acknowledgments}

The authors thank Ms. F. Zhang for her work in the clean room to fabricate the electrodes. We would like to acknowledge Dr. Joey Kish for providing us the access to the Walter W. Smelter Corrosion Laboratory and Mr. M. Taheri for his assistance in electrochemical measurements.

\section{References}

1. S. Cogan, Annu. Rev. Biomed. Eng., 10, 275 (2008).

2. W. M. Grill, IEEE EMBS Conf., 2369 (2009).

3. D. K. Peterson, M. L. Nochomovitz, T. A. Stellato, and J. T. Mortimer, IEEE Trans. Biomed. Eng., 41(12), 1127 (1994).

4. J. D.Weiland, W. Liu and M. S. Humayun, Annu. Rev. Biomed. Eng., 7, 361 (2005).

5. F. Zeng, S. Rebscher, W. Harrison, X. Sun and H. Feng, IEEE Reviews in Biomed. Eng., 1, 115 (2008).

6. J. D. Weiland, D. J. Anderson and M. S. Humayun, IEEE Trans. on Biomed. Eng., 49(12), 1574 (2002).

7. K. Singh, F. J. R. Richmond, and G. E. Loeb, IEEE Trans. on Rehab. Eng.,8(3), 276 (2000).

8. B. D. Ratner, Biomaterials science,p. 355, Elsevier Academic Press, $2^{\text {nd }}$ edition USA (2004).

9. D. R. Merrill, In Elias Greenbaum, David Zhou, and Elias Greenbaum, editors, Implantable Neural Prostheses 2, Biological And Medical Physics Biomedical Engineering, p. 85-138, Springer New York, (2010).

10. N. Tandon, C. Cannizzaro, E. Figallo, J. Voldman, G. V. Navokovic, Proceedings of the $28^{\text {th }}$ IEEE EMBS Conf., New York (2006).

11. S. H. Lee, J. H. Jung, Y. M. Chae, J. F. Suh, J. Y. Kang, J. Micromech. Microeng., 20, 035015(2010).

12. E. T. McAdams, J. Jossinet, R. Subramanian, R. G. E. McCauley, Proceedings of the $28^{\text {th }}$ IEEE EMBS Conf., New York (2006).

13. Y. Shacham-Diamond, S. Krylov, T. Shmilovich, R. O. Almog, N. Fishelson, Y. Sverdlov, I. Torchinsky, G. Rosenman, A. Inberg and O. Berkh, ECS Trans., 23(1), 243 (2009).

14. F. J. Rodriguez, D. Ceballos, M. Schuttler, A. Valero, E. Valderrama, T. Stieglitz, X. Navarro, Neuroscience Methods, 98, 105 (2000). 
15. K. Takata, A. Pham, $6^{\text {th }}$ IEEE Conf. on Polymers and Adhesives in Microelectronics and Photonics, Tokyo (2007).

16. S. Negi, R. Bhandari, L. Rieth, F. Solzbacher, Sensors and Actuators B: Chemical, 137, 370 (2009).

17. A. Hung, I. B. Goldberg, J. W. Judy, Implantable Neural Prostheses 2 Biological and Medical Physics, Biomedical Engineering, Springer-Verlag New York (2010).

18. C. M. A. Brett, ECS Trans., 13(13), 67 (2008).

19. J. Wolpaw, J. Motor Behavior, 42(6), 351 (2010).

20. A. E. Schultz, T. A. Kuiken, Phys. Med. Rehab., 3(1), 55 (2011).

21. P. Brunner, L. Bianchi, C. Guger, F. Cincotti, and G. Schalk, J. Neural Eng, 8(2), 025001 (2011).

22. J. Kubánek, K. J. Miller, J. G. Ojemann, J. R. Wolpaw, and G. Schalk, J. Neural Eng, 6(6), 066001 (2009). 